\title{
Les gisements de nappes profondes en France : concept, spécificités, état des connaissances
}

\author{
par Jean-Pierre Comte
}

du BRGM, Service géologique national, Centre thématique Eau, Montpellier

\section{NAPPE PROFONDE OU CONTRIBU- TION À LA THÉORIE DE LA RELATI- VITÉ... !}

Définir l'objet de cette journée est un préalable indispensable. Les nappes dites profondes occupent une place particulière dans le vaste monde de l'eau souterraine : c'est cette place que nous nous efforcerons de définir.

Tout d'abord si nous parlons de « nappe ", nous considérons implicitement un système aquifère continu bien identifié en tant que tel, c'est-à-dire un système fini (dans des limites à préciser... d'autant plus difficilement qu'elles sont profondes !). Ce concept exclut donc les gisements discontinus ou ponctuels notamment des milieux fissurés, fracturés, filoniens. Certains de ces gisements sont pourtant parfois profonds comme en témoignent nombre de systèmes karstiques ou fissurés et de forages hydrothermaux (à plus de $1000 \mathrm{~m}$ notamment): on ne pourra pas les ignorer, mais leur identification relève d'une autre échelle, beaucoup plus locale.

«Profond/pas profond » c'est a priori comme « grand/ petit », « beau/pas beau », « cher/pas cher »!... pour ne pas rester dans une subjectivité risquant de donner libre court à des débats qui n'avancent à rien, il faut se rattacher à une échelle de valeur. «Beau » ne le permet pas. « Grand, cher... profond » le permettent.

L'échelle de valeur la plus fiable est bien sûr la profondeur mesurée. Mais cette échelle varie selon les utilisateurs, et dans le temps!

- ce qui paraissait profond autrefois, peut aujourd'hui apparaître ordinaire, sinon peu profond...,

- ce qui paraît profond pour un foreur d'eau paraît dérisoire pour un foreur pétrolier...

Par ailleurs, toutes les profondeurs de gisement sont loin d'être mesurées!

Dès lors peut-on constater deux configurations pour parler de «nappe profonde ":

- les nappes dites profondes dans l'absolu parce qu'accessibles au-delà d'une « certaine » profondeur,

- les nappes « les plus profondes " d'un système multicouche ou particulièrement puissant, ou plus simplement «dites profondes » par rapport à un autre système dit « superficiel » ou peu profond.

\subsection{Nappe « absolument » profonde}

La référence est la profondeur absolue d'accès au toit de la formation aquifere : mais comment, à travers les chiffres, apprécier la notion de profondeur? Plusieurs démarches sont envisageables :

a) Une première façon d'apprécier la profondeur absolue pourrait être de lui faire correspondre des techniques de forage d'eau. Ainsi, dans certains pays anglophones, la perception courante de la différence entre "shallow well " et "deep well » est relative au mode d'exécution : puits (creusés à la main) pour les premiers, forage pour les seconds. La profondeur absolue en est une conséquence étant entendu qu'on utilisera le forage pour atteindre des profondeurs inaccessibles au creusement manuel des puits. Mais force est de constater que l'évolution des technologies repousse toujours plus loin (plus profond) les performances des machines (ex.: le forage à l'air longtemps limité à la centaine de mètres, frise aujourd'hui les $1000 \mathrm{~m} \ldots$..).

b) Une seconde façon de faire serait de considérer qu'une nappe est profonde parce qu'elle n'est accessible que par des forages nettement plus profonds que ceux couramment pratiqués pour l'exploitation de l'eau souterraine.

Au-delà d'un ordre de grandeur de $200 \mathrm{~m}$, on est dans ce cas de figure.

On constate en effet que sur un échantillon de près de 86000 forages d'eau recensés et renseignés dans la Banque de données du Sous-Sol (BSS). $96 \%$ d'entre eux ne dépassent pas cette profondeur (91\% ne dépassent même pas $100 \mathrm{~m}$ !) (voir fig. l).

Ces chiffres montrent l'arbitraire d'une limite à $200 \mathrm{~m}$, plutôt que 100, 200 ou 500 !

c) Une démarche plus « synthétique » pourrait consister à dénombrer non plus les forages, mais les «nappe » en fonction de leur profondeur d'accès. L'exercice est pratiquement difficile car ces nappes ne se situent jamais à profondeur quasi constante (sauf dans un espace donné) : rares sont les plus « profondes » qui n'affleurent pas à l'amont du système (ex. : aquifères profonds des Bassins parisien et aquitain). Dès lors ce ne serait que les profondeurs maximales d'accès 


\begin{tabular}{|c|c|c|c|c|}
\hline Profondeur $(\mathrm{m})$ & 0 à 50 & 50 à 100 & 100 à 200 & 200 à $>2000$ \\
\hline Nbre de forages & 64700 & 15000 & 4400 & 1460 \\
\hline$\%$ & 74 & 17 & 5 & 4 \\
\% cumulé & 74 & 91 & 96 & 100 \\
\hline
\end{tabular}

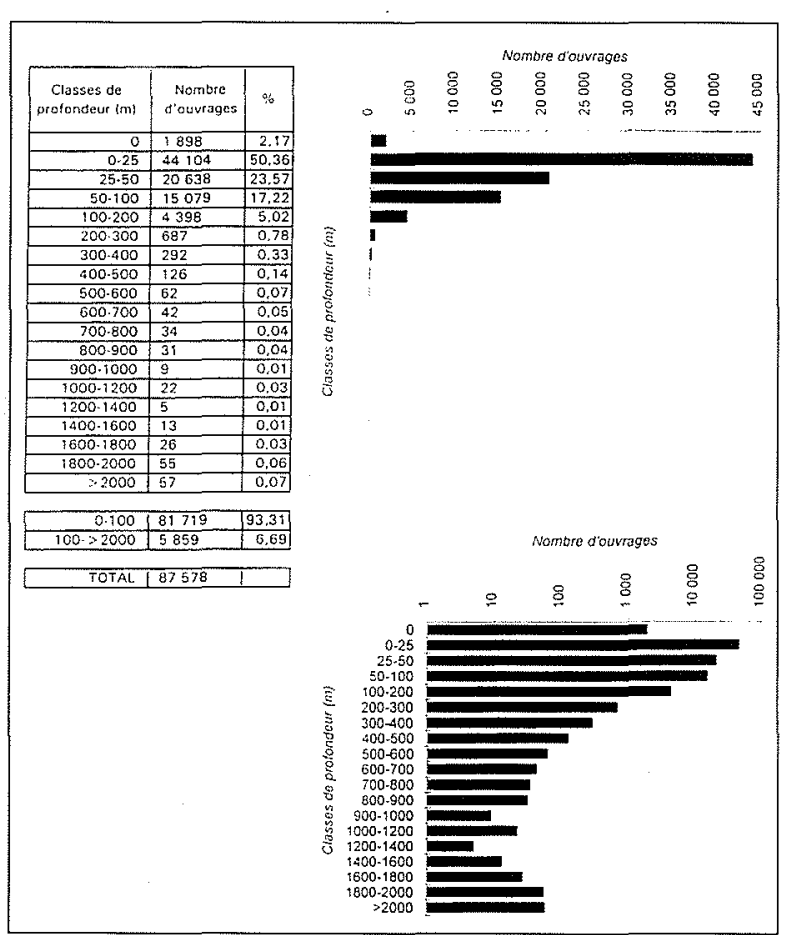

1. Ouvrages d'exploitation d'eau répartis en classes de profondeur. (échantillon de 85550 forages extraits de la BSS.)

qu'il faudrait traiter. Mais lesquelles : celles reconnues ou celles supposées?..

Les connaissances apportées par les forages à plus de 200 $\mathrm{m}$ sont bien trop peu nombreuses (voir fig. I), fragmentaires et ponctuelles pour permettre des évaluations à des niveaux de précision comparables à ceux des nappes moins profondes. Ces ouvrages constituent cependant de précieux étalonnages au raisonnement géologique. La connaissance des nappes profondes ne progressera qu'avec leur nombre, comme souvent en hydrogéologie.

Sur plus de 500 systèmes aquifères identifiés au niveau national, une cinquantaine ont des profondeurs maximales d'accès qui atteignent ou dépassent $200 \mathrm{~m}$. Le tableau ciaprès en présente certaines de leurs caractéristiques.

Cette cinquantaine de systèmes (ou « nappes conti-nues ») se répartit ainsi en fonction des profondeurs maximales d'accès.

Il est évident que pour mesurer l'importance réelle de ces nappes, il faudrait ajouter la prise en compte de leur superfi- cie, leurs ressources, leur taux d'exploitation; mais cela dépend des "états des connaissances » qui seront évoqués ci-dessous.

On constate que les profondeurs d'accès de $75 \%$ des systèmes localisés à plus de $200 \mathrm{~m}$ ne dépassent pas $1000 \mathrm{~m}$.

La limite quasi-asymptotique à plus de $2000 \mathrm{~m}$ semble donc davantage correspondre à une réalité physique, qu'à une limite de connaissance ou d'exploitabilité technique. Ceci bien sûr indépendamment des « gisements » profonds particuliers et localisés qui peuvent être exploités en tant que ressource thermo-minérale.

1.2 Nappe « relativement » la plus profonde d'un système multicouche ou par rapport à un système superficiel

Dans le premier cas il peut s'agir des niveaux les plus profonds d'un système unique particulièrement profond. Ces niveaux peuvent en effet avoir des caractéristiques particulières qui justifient un mode d'exploitation et des usages spécifiques : caractéristiques hydrodynamiques de l'aquifère, paramètres physico-chimiques notamment la qualité alimentaire pour l'AEP ou la température en vue de valorisation énergétique par exemple.

Dans le second cas il s'agit d'une dénomination relative pour identifier la nappe inférieure d'un système bicouche. Ce cas est très fréquent et sort donc de notre contexte puisque les profondeurs d'accès sont couramment de l'ordre de grandeur de celles d'autres systèmes réputés non profonds, notamment lorsqu'il s'agit de nappes alluviales superficielles (ex.: « nappe profonde » de l'Astien du Languedoc, qui n'est qu'à peine à une centaine de mètres sous les nappes alluviales).

\section{LES GISEMENTS D'EAU SOUTER- RAINE PROFONDE DE FRANCE}

Pour partir à la découverte des nappes profondes du territoire métropolitain, plusieurs chemins sont possibles :

- la géographie, du Nord au Sud, région par région à la satisfaction des opérateurs régionaux précisément,

- la géométrie, de la moins profonde à la plus profonde : mais la variabilité, outre les imprécisions à grande profondeur pour un même système, empêcherait toute rigueur dans une telle démarche,

- reste la géologie qui offre l'avantage d'un langage commun devenu dorénavant quasiment incontestable. Une telle

Répartition des systèmes aquifères en fonction de leur profondeur maximale d'accès.

\begin{tabular}{|c|c|c|c|c|c|}
\hline Prof. max. (m) & $<500$ & 500 à 1000 & 1000 à 1500 & 1500 à 2000 \\
\hline Nbre de unappes " \% & 45 & 30 & 10 & 9 \\
\hline$\%$ cumulé & 45 & 75 & 85 & 94 \\
\hline
\end{tabular}


approche suppose toutefois d'être parfaitement conscient de ses limites :

- selon sa situation, un même niveau stratigraphique peut être profond, ou non, peut même affleurer à tel endroit avant de plonger profondément : c'est un cas très fréquent ;

- ce même niveau pourra avoir des faciès différents selon la profondeur (présence de sédimentation, d'érosion, de karstification, de modifications structurales au fil du temps);

- en conséquence, il n'y a pas systématiquement correspondance entre niveau stratigraphique et propriétés aquifères des formations. C'est vrai et presque « visible » pour les plus superficielles : ce l'est d'autant plus, et d'autant moins appréhensible pour les plus profondes.

On s'efforcera donc de régionaliser l'approche géologique pour mettre en évidence les variations de caractères (profondeur, épaisseur, productivité, qualité de l'eau...) des différentes formations dès lors que leur extension le justifie.

Pour plus de commodité, nous explorerons le sous-sol de notre territoire en quatre étapes, à quatre niveaux successifs correspondant donc à une approche purement géologique des systèmes aquifères dont les profondeurs moyennes d'accès atteignent et dépassent 200 mètres (voir tableau l).

Tableau 1. - Inventaire national des ressources en eau souterraine profondes.

Premier sous-sol : systèmes aquifères du Tertiaire

\begin{tabular}{|c|c|c|c|c|c|c|c|c|c|c|c|c|}
\hline \multirow[b]{2}{*}{1} & \multirow{2}{*}{$\frac{N \cdot S . A}{225}$} & \multirow{2}{*}{$\frac{\text { Rigion }}{\text { L-R }}$} & \multirow{2}{*}{$\begin{array}{l}\text { Dénomination } \\
\text { multicotuche du Roussition }\end{array}$} & \multirow{2}{*}{ Géologie } & \multicolumn{2}{|c|}{ Profondeur } & \multicolumn{2}{|c|}{ Epaisseur } & \multirow{2}{*}{ Exploitation } & \multirow{2}{*}{$\frac{\text { Modele }}{\text { out }}$} & \multirow{2}{*}{$\frac{\text { Qualité }}{\text { borme }}$} & \multirow{2}{*}{$\frac{\text { Observations }}{\text { coppl }}$} \\
\hline & & & & & 30 & 250 & 100 & 250 & & & & \\
\hline 2 & $556 b$ & $L \cdot R$ & $\begin{array}{l}\text { gres des garrigues du sud-Vidourle et five droile } \\
\text { du bassin de Castries Sommieres }\end{array}$ & miocene & 50 & $>200$ & 30 & 100 & AEP & & & \\
\hline 3 & 235 & AOUI & grès of calc. et sabtes miocènes d'Aquilfalne & $\begin{array}{l}\text { hélwatien } \\
\text { aquitarton }\end{array}$ & 10 & $>200$ & 10 & 100 & $\begin{array}{c}\text { pluss.10zaines } \\
\text { M mitan }\end{array}$ & & & coptr \\
\hline \multirow[t]{3}{*}{4} & 212 & RH-A & sables el molasse de Bresse & miocene & 180 & 360 & 10 & 150 & peu & & $\operatorname{dance}$ & capts \\
\hline & $212 a$ & BOU & miocene de Bresse buhannaise & miocene & & 400 & & 100 & très peu & & & capst \\
\hline & $212 b$ & BOU & miocene de Dijon-Chabn & miocene & 100 & 400 & & 100 & très peu & & & capot \\
\hline 5 & 228 & PACA & molasso ef sabios mboctnas du Comtet & $\begin{array}{l}\text { miocène } \\
\text { burdigalen }\end{array}$ & 150 & 300 & 50 & 150 & pes & & $\operatorname{sen} \theta \sin$ & captr \\
\hline 6 & $25-27$ & CEN & calcaires miocenes de Beauce et de Sologne & aquitanien & 60 & 200 & & 100 & oui & partiel & Fer & copter en Sologne \\
\hline 7 & 230 & AOUI & catcatres of gros olgocenes d'Aquilahe & olligocene & 20 & 600 & 50 & 500 & $\begin{array}{c}\text { phus. 10zahes } \\
M \text { mifan }\end{array}$ & partiet & Dame & coon: \\
\hline \multirow[t]{2}{*}{8} & 214 & AQUI & $\begin{array}{l}\text { mulficouche calcaire+sables } \\
\text { inframolassique d'Aquitaine }\end{array}$ & Éocène inf. & 50 & $>1000$ & 50 & 300 & $\begin{array}{c}\text { plus. } 10 \text { zaines } \\
M \mathrm{~m}^{3 / a n}\end{array}$ & partiel & bome & capss \\
\hline & 214 & M-Py & $\begin{array}{l}\text { sables infra-molassiques éocènes } \\
\text { de Midi-Pyrénées }\end{array}$ & $\begin{array}{c}\text { éocène inf. } \\
\text { ypresien }\end{array}$ & 100 & 800 & $<20$ & $>100$ & env. $10 \mathrm{M} \mathrm{m} 7 / \mathrm{an}$ & Ouest & bome & capss \\
\hline 9 & $557 a 1$ & L-R & calcates du Mhervots & écerne & 10 & $>300$ & 50 & 150 & pers & & vocson. & \\
\hline 10. & 557 a1 & L-R & bassin de Castelnaudary & éocéne inf. & 50 & $>500$ & 20 & 150 & peu & & AEP Casten & \\
\hline 11 & $557 a 2$ & $L-R$ & $\begin{array}{l}\text { calcaires du Carcassonnais of de Lezignan- } \\
\text { Corbieres }\end{array}$ & $\begin{array}{c}\text { écéne } \\
\text { +jurassique } \\
\end{array}$ & 10 & $>500$ & 50 & 500 & pou & & & \\
\hline 12 & $142 \mathrm{a}$ & $L-R$ & bassin calc. éocène de St Martin de Londres & Iutètien & 20 & 300 & 50 & 100 & & & bonne & \\
\hline 13 & 233 & AQUI & dolomios at cateater des Landes & $\begin{array}{l}\text { dano- } \\
\text { palécoene }\end{array}$ & 50 & $>2000$ & 100 & 300 & taith & & stotatire & contil \\
\hline
\end{tabular}

Deuxième sous-sol : systèmes aquifères du Crétacé

\begin{tabular}{|c|c|c|c|c|c|c|c|c|c|c|c|c|}
\hline \multirow[b]{2}{*}{14} & \multirow{2}{*}{$\begin{array}{c}\frac{N}{N} \text { S.A. } \\
\begin{array}{c}44-44 \times-46- \\
47-49\end{array} \\
\end{array}$} & \multirow{2}{*}{$\frac{\text { Region }}{\mathrm{CEN}}$} & \multirow{2}{*}{\begin{tabular}{|l|} 
Dénomination \\
crale crétac de Salogne \\
\end{tabular}} & \multirow{2}{*}{$\begin{array}{c}\text { Géologie } \\
\text { sentrontert } \\
\text { turonters }\end{array}$} & \multicolumn{2}{|c|}{ Profondeur } & \multicolumn{2}{|c|}{ Epaisseur } & \multirow{2}{*}{$\frac{\text { Exploitation }}{\text { out }}$} & \multirow[t]{2}{*}{ Modèle } & \multirow[t]{2}{*}{ Qualité } & \multirow{2}{*}{$\frac{\text { Observation }}{\text { sentaptit }}$} \\
\hline & & & & & 100 & 300 & 1503 & 300 & & & & \\
\hline 15 & 231 & AOUI & $\begin{array}{l}\text { catcaires et dolomies du crétacé supérieur } \\
\text { d'Aquitaine }\end{array}$ & $\begin{array}{l}\text { maestrich.+ } \\
\text { campanien }\end{array}$ & 50 & $>1000$ & $100 \quad 2$ & 200 & forte & parties & rome & capur \\
\hline \multirow[t]{2}{*}{16} & 205 & CEN & sables du cénomanten de nord-Touraine & crétaces sup. & 100 & 200 & 50 & 100 & intense & & duro & copist \\
\hline & 205 & Pdil & sabtos du cenomanten de sud-Tounatne & créteces sup. & 100 & 300 & 50 & 80 & forte & out & & capn \\
\hline 17 & 215 & AQUI & sables cénomaniens et calcaires du crét. sup. & crétacé sup. & 700 & 2400 & \multicolumn{2}{|c|}{ moy. 400} & intense & oui & bonne & capot \\
\hline 18 & 219 & RHA & calcaires crátecé du Dauphin'́ / Vercors & crét. inf. & & 250 & \multicolumn{2}{|l|}{ varkatio } & & & $F_{\theta}$ & coptit \\
\hline 19 & 220 & PACA & calcaires urgoniens du Tricastin (discontinus) & crét. inf. & 400 & 1000 & 2004 & 400 & & & & capor \\
\hline 20 & 229 & PACA & cakcakes "urgontons" du bessin d'Apt - Vaucture & crét. irf. & 500 & 1000 & \multicolumn{2}{|l|}{ variato } & peu & & borne & coptif \\
\hline 21 & 221 & PACA & sables, gres et calcaires cretace du Comtat & cretace & 150 & 900 & 400 & 500 & & & & captr \\
\hline 22 & 222 & PACA & cakcalres urgoniens de Berre-Gardanne & crét. inf. & 50 & 500 & 400 & 500 & oul & & & 20014 \\
\hline 23 & 149 & L-R & $\begin{array}{l}\text { calcaires utgoniens du synclinal de } \\
\text { Gardannanque }\end{array}$ & crèt. inf. & 20 & 500 & 100 & 400 & inrigation & & bane & \\
\hline \multirow[t]{3}{*}{24} & $204 a$ & IdF & sables verts de ralloten dille de Franco & crét. inf. & 200 & 700 & 30 & 45 & out & & Fer & coptr \\
\hline & $204 a$ & CEN & sables verts de ralbien du Centre & cret. inf. & 10 & 600 & 0 & 35 & out & & dro & cow \\
\hline & 213 & BOU & sables de ratbion de Bourgogne & cretece he. & 50 & 600 & & & ous & & & $\cos$ \\
\hline \multirow[t]{2}{*}{25} & $204 \mathrm{~b}$ & PIC & sables du néocomien & $\begin{array}{l}\text { barrémien- } \\
\text { hauterimen }\end{array}$ & 120 & 250 & 15 & 30 & oui & non & $\begin{array}{l}\text { saie vers } \mathrm{NWW} \text { : } \\
0.5 \rightarrow 3 \text { gi NaCl }\end{array}$ & capt \\
\hline & $204 \mathrm{~b}$ & IdF & sables du neocomien d'lle de France & $\begin{array}{l}\text { barremien- } \\
\text { hauterivien }\end{array}$ & 400 & 900 & & 150 & & & Fer & sapar \\
\hline 26 & $144 a$ & L-R & bassin calcake de Qulluan & cretecé inf. & 50 & $>200$ & 150 & 250 & & & Dorne & \\
\hline
\end{tabular}


Troisième sous-sol : systèmes aquifères du Jurassique

\begin{tabular}{|c|c|c|c|c|c|c|c|c|c|c|c|c|}
\hline & $N^{*}$ S.A. & Région & Dénomination & Geologie & Profor & ndeur & Epaiss & seur & Exploitation & Modele & Qualite & Observations \\
\hline \multirow[t]{6}{*}{27} & 206 & N.PdC & $\begin{array}{l}\text { jurassique sup. du Nord } \\
\text { (calcaire karstifie) }\end{array}$ & $\begin{array}{l}\text { kimmer. } \\
\text { oxtardien }\end{array}$ & 100 & 350 & & 50 & & & $\begin{array}{c}7 \text { gl NaG à } \\
\text { Arrens }\end{array}$ & $\begin{array}{l}\text { Bouromass et sudt } \\
\text { Picarde }\end{array}$ \\
\hline & 206 & CEN & $\begin{array}{l}\text { urassique sup. du Centre } \\
\text { (calcaire karstifie) }\end{array}$ & $\begin{array}{c}\text { kimmendg. } \\
\text { iustianen }\end{array}$ & 0 & 1000 & & 500 & & & sure & capat \\
\hline & 206 & IdF & $\begin{array}{l}\text { jurassique sup.(malm) ditle de france } \\
\text { (calcaire karstrie) }\end{array}$ & $\begin{array}{l}\text { kimmeridg. } \\
\text { iusilanen }\end{array}$ & 1200 & 1800 & & 500 & & & 5 a 6 grn sutatee & capor \\
\hline & 206 & Ch-A & $\begin{array}{l}\text { jurassique sup. de Champagne } \\
\text { (calcaire karstine) }\end{array}$ & $\begin{array}{l}\text { kimmeri- } \\
\text { dgien }\end{array}$ & 350 & 1300 & & 500 & & & are & capor \\
\hline & 206 & BOU & $\begin{array}{l}\text { calcaires du Barrois (jurassique sup. de } \\
\text { Bourgogne) }\end{array}$ & malm & 100 & 200 & & 90 & & & & capot \\
\hline & 206 & LOR & calcaires rauraciens de Lorraine (jur.sup.) & oxfordien & 50 & 200 & 50 & 150 & peu & & bome & capt1 \\
\hline \multirow[t]{8}{*}{28} & 207 & PIC & dogger de Picardla & $\begin{array}{l}\text { bathonten } \\
\text { bajocien }\end{array}$ & 100 & 900 & 50 & 100 & & & 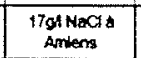 & capti \\
\hline & 207 & IdF & dogger ditle de France & $\begin{array}{l}\text { callowiten } \\
\text { baltionten }\end{array}$ & 1500 & 2000 & & 300 & géplhermbe & oul(1986) & $200300 \mathrm{gt}$ & cooted \\
\hline & 207 & PdL & dogger de ka Sartho & jutass, moy. & 100 & 200 & & & oui & & $\sin v \operatorname{rest}$ & $\cos 1$ \\
\hline & 207 & CEN & dogger du Berry & $\begin{array}{c}\text { callorien } \\
\text { bethonion }\end{array}$ & 100 & 250 & & 100 & & & & $\operatorname{coptif}$ \\
\hline & 207 & $\mathrm{P}-\mathrm{Ch}$ & doggar du Nord tu seull du Paitot & jurass.moy. & 250 & 500 & & & oui & out 93 & $\begin{array}{l}\text { Salbe vers le } \\
\text { Nard }\end{array}$ & cotf \\
\hline & 207 & ChinA & dogger de Champagne & bath-callow. & 600 & 1600 & & 300 & & & & caplif \\
\hline & 207 & LOR & dogger de Lomaine & bath-caliow. & 50 & 300 & 100 & 150 & $\begin{array}{c}\text { oui } \\
\text { si peu profond }\end{array}$ & & borme & 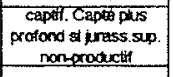 \\
\hline & 207 & BOU & dogger de Bourgogne & bath-callow. & 80 & 300 & & 90 & & & & $\cos 1$ \\
\hline 29 & 217 & AQU: & calcaires karstifie du jurassique d'Aquitaine & $\begin{array}{l}\text { malm+ } \\
\text { dogger }\end{array}$ & 1000 & 2500 & 50 & 200 & oul & & $\begin{array}{l}\text { Donne en } 24 \\
\text { sale alleurs }\end{array}$ & $\begin{array}{l}\text { capot. Geothermeen } \\
\text { Gironde }\end{array}$ \\
\hline 30 & 145 & L-R & syncinal des Fonoulledes & $\begin{array}{l}\text { Jur.tcrét. } \\
\text { Infér. }\end{array}$ & 20 & $>300$ & 200 & 500 & peis & & bomo & \\
\hline 31 & 166 & PACA & bassin du Beausset & jutassique & 50 & 1000 & 200 & 400 & & & vanabteprof. & \\
\hline 32 & 706 & PACA & bassin jurassique de Marselle-Allauch & jurarsiques & 50 & 300 & 200 & 300 & & & vartablapof. & \\
\hline 33 & 223 & PACA & calcaire jurassique de vatensole & jurass.sup. & 100 & $>1000$ & 200 & 300 & & & vanableprof. & capait \\
\hline 34 & $557 c$ & LR & cakcalre jur. sup. du pli ouest de Nontpellior & matm & 50 & $>1000$ & 100 & 500 & pert & oul & roseser. & 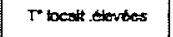 \\
\hline 35 & 224 & PACA & $\begin{array}{l}\text { karst profond de la moyenne Durance el calcaire } \\
\text { jurassique d'Aix en } P C \theta\end{array}$ & jurassique & 50 & 1500 & & 500 & & & & captf \\
\hline 36 & $143 b$ & L-R & fossé calcaire de Montizazh-Gigean & jurassique & 50 & $>300$ & 3001 & 1500 & peu & out & borne & \\
\hline 37 & 208 & Ch-ALLOR & catcaires et grès héttangizn - sinémurien & lias & 25 & & & & oui & & & capbs \\
\hline 38 & $\begin{array}{l}147+148+ \\
218+607 \\
\end{array}$ & RHAA & calcalres du sud-est de IArdiche & thas & 130 & 300 & & & & & & \\
\hline 39 & 232 & AQUI & dolomies et gres du lias d'Aquitaine & lias - infratias & 50 & $>2000$ & 100 & 200 & & & rome & capst \\
\hline 40 & 241 & $\mathrm{P} \cdot \mathrm{Ch}$ & cakcaires de Pinfre-toarcien du sud-Poilou & hass & 100 & 500 & 50 & 100 & oui & & $\begin{array}{c}\begin{array}{c}\text { saboe a partí do } \\
300 \mathrm{~m}\end{array} \\
\end{array}$ & 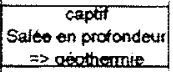 \\
\hline 41 & $\begin{aligned} & 138 \mathrm{~b} \\
&+ 139 \mathrm{ab} \\
&\end{aligned}$ & $L-R$ & $\begin{array}{l}\text { calcaires el dolomies karstifiés des causses } \\
\text { Sauvet. Méjean. Noir }\end{array}$ & lias int. & 100 & $>500$ & 100 & 200 & peu el par sources & & tome & Litres et untherables \\
\hline
\end{tabular}

Quatrième sous-sol : systèmes aquifères du Trias et du Primaire

\begin{tabular}{|c|c|c|c|c|c|c|c|c|c|c|c|c|}
\hline & $N^{*} S . A$ & Region & Dénomination & Géologie & Profo & ideur & Epaiss & seur & Exploitation & Modele & Qualité & Observations \\
\hline \multirow[t]{2}{*}{42} & 210 & LOR & grès du trigs inferieur de Lomaine & $\begin{array}{c}\text { triast } \\
\text { muschetal }\end{array}$ & 10 & $>800$ & & 500 & oui & ouli & \begin{tabular}{|c|} 
sole vers foused \\
i prol $>800 \mathrm{~m}$
\end{tabular} & eapir \\
\hline & 211 & FR-C & grès du trias inférieur du sud du BP & trias inf. & & & 200 & 500 & oui & & 8 sow wors $\mathrm{NW}$ & capti \\
\hline 43 & 705 & PACA & bassin de Brignoles & jur.int.+trias & 200 & 900 & 100 & 200 & & & & \\
\hline 44 & $202^{-}$ & NPdC & cakcalos fissurés cartontheres du Nord & dixantion & 50 & 1000 & & 85 & $\begin{array}{c}\text { Intenso } \\
\text { au N de Lithe }\end{array}$ & oui & 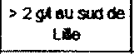 & coptf \\
\hline 45 & 227 & $L-R$ & calcaires primaires du lodevors & cambrien & 10 & $>500$ & 100 & 300 & geothermie & & Y elevere & \\
\hline
\end{tabular}

Source : BRGM Thématique Eau 
Au premier sous-sol, quasiment tous les étages du Tertiaire sont représentés par des systèmes «les moins profonds des profonds $\gg$ :

- Pliocène multicouche du Roussillon, Miocène du Languedoc mais aussi de Beauce, de Sologne et d'Aquitaine, pouvant atteindre $250 \mathrm{~m}$ de profondeur et très exploités ;

- sables et molasse miocène de Bourgogne, de Bresse et du Comtat jusqu'à 300 à $400 \mathrm{~m}$ de profondeur, assez peu exploités;

- calcaires et grès sableux oligocènes (jusqu'à $600 \mathrm{~m}$ ) et éocènes (à plus de $1000 \mathrm{~m}$ ) d'Aquitaine, en continuité avec l'inframolassique de Midi-Pyrénées (jusqu'à $800 \mathrm{~m}$ ), tous assez fortement sollicités pour l'AEP — près de $160 \mathrm{Mm}^{3}$ / an - et relativement bien connus ;

- plusieurs bassins bien circonscrits du Languedoc-Roussillon offrent des réservoirs éocènes jusqu'à $500 \mathrm{~m}$ de profondeur et très peu exploités (Castelnaudary, Minerve, Carcassonne, Corbières...);

- enfin, à la base de ce premier sous-sol, il faut citer le dano-paléocène d'Aquitaine, puissant, pouvant dépasser les $2000 \mathrm{~m}$ de profondeur mais peu exploité, d'autant que la qualité de l'eau y est aléatoire.

Au deuxième sous-sol, le Crétacé supérieur est représenté par différents faciès :

- la craie de Sologne (jusqu'à $300 \mathrm{~m}$ ) et sables cénomaniens de Touraine : jusqu'à $300 \mathrm{~m}$ et très exploités ;

- les puissantes et profondes séries d'Aquitaine, des calcaires et dolomies du Maestrichtien aux sables cénomaniens et calcaires qui, avec des épaisseurs de 200 à $400 \mathrm{~m}$ peuvent dépasser $1000 \mathrm{~m}$ de profondeur pour les premiers, et $2400 \mathrm{~m}$ pour les seconds.

Deux formations sont datées du Crétacé inférieur :

- les calcaires massifs « urgoniens » du Sud-Est (couloir rhodanien et Provence), compte tenu d'une tectonique préalpine très intense, peuvent constituer des réservoirs puissants (quelques centaines de mètres), de profondeurs variables allant de 100 à plus de $1000 \mathrm{~m}$ de profondeur (dans le Tricastin). Ils peuvent être localement exploités et parfois connus en tant que gisements thermo-minéraux (ex. : Gréouxles-Bains) ;

- la vaste et célèbre formation aquifère des sables de l'Albien dont l'extension va du Nord-Ouest du Bassin parisien à la Bourgogne en passant par l'Ile de France où ils sont bien connus et parfois exploités, et la région Centre. Leur profondeur maximale est de 600 à $700 \mathrm{~m}$ et leur salinité augmente vers le Nord-Ouest, pouvant passer de 0,5 à $3 \mathrm{~g} / \mathrm{l}$ de $\mathrm{NaCl}$. Ils surmontent les sables du Néocomien en Ile de France.

Au troisième sous-sol, règnent les puissants calcaires du Jurassique, parfois karstifiés en tête de séries (malm). On distingue encore une fois les petits systèmes compartimentés et isolés du Sud-Est, des grandes formations des bassins parisiens et aquitains.

- Concernant ces derniers, il s'agit

- d'une part des calcaires du Jurassique supérieur ou malm, plus précisément kimmeridgiens (lusitaniens d'Ile de France et du Centre, oxfordiens moyen et supérieur du Nord, rauraciens de l'Est...) : ils affleurent au pourtour du bassin (Lorraine, région Centre et Ouest du Bassin) mais dépassent $1000 \mathrm{~m}$ de profondeur dans le Centre, la Champagne et l'Ile de France (1 200 à $1800 \mathrm{~m}$ ). Dans ces régions, ils sont également les plus puissants (de l'ordre de $500 \mathrm{~m}$ ). Leur salinité dépasse généralement les $5 \mathrm{~g} / \mathrm{l}(7 \mathrm{~g} / \mathrm{l}$ de $\mathrm{NaCl}$ à Amiens) ;

- d'autre part des calcaires du Jurassique moyen ou Dogger, plus précisément calloviens, bathoniens, bajocioens. Ils s'étendent eux aussi de la Picardie au Nord du Seuil du Poitou, à la Lorraine et à la Bourgogne. Leur profondeur minimale d'accès va d'une centaine de mètres à 600 en Champagne et 1500 en Ile de France, où on les trouve encore à $2000 \mathrm{~m}$ pour des exploitations géothermiques. Cet aquifere offre localement des débits de plus de $250 \mathrm{~m}^{3} / \mathrm{h}$ à $80^{\circ} \mathrm{C}$ de température... L'eau est très salée, chlorurée sodique ;

- on retrouve cette puissante série de calcaires aquifères fissurés dans le Bassin aquitain, entre 1000 et $2500 \mathrm{~m}$ de profondeur: Exploités en Dordogne pour l'AEP, ils n'ont plus qu'un usage géothermique en Gironde.

- Concernant les systèmes compartimentés du Sud-Est, jurassique supérieur et moyen, ils correspondent à de petits bassins en Provence (Beausset, Allauch, Durance, Valensole) ou en Languedoc (Montpellier, Gigean...). Il donnent lieu localement à du thermalisme (Digne, Aix-en-Provence).

A la base de troisième sous-sol, des systèmes aquifères du Lias sont identifiés au Nord de la Champagne et de Lorraine (calcaires et grès hettangien-Sinémurien), dans le SudEst de l'Ardèche, les Causses, et bien sûr dans le Bassin aquitain (grès et dolomies) jusqu'à plus de $2000 \mathrm{~m}$ de profondeur. L'infratoarcien du sud du Seuil du Poitou est certainement un aquifère prometteur de 100 à $500 \mathrm{~m}$ de profondeur:

Au quatrième sous-sol, on trouve deux types de formation:

- les formations gréseuses du Trias, avec les grès du Trias inférieur de Lorraine, des Vosges et du Sud du Bassin parisien. S'ils affleurent sur le flan ouest des Vosges, ces grès plongent très vite. Au droit de Nancy, ils atteignent $800 \mathrm{~m}$ de profondeur et deviennent trop salés vers l'Ouest pour être exploitables. Au Sud du Bassin parisien, ils sont aussi puissants (jusqu'à plusieurs centaines de mètres) mais deviennent salés vers le Nord-Ouest.

- les formations calcaires fissurées du Primaire essentiellement représentées dans le Nord: calcaire carbonifère peu profond en Belgique et s'approfondissant vers l'Ouest, en France, jusqu'à environ $1000 \mathrm{~m}$. Ils sont bien exploités au Nord de Lille, tandis que leur salinité augmente vers le Sud.

Enfin, au Sud de la France, les calcaires cambriens du Lodévois (Haut Languedoc) sont exploités pour la géothermie, à plusieurs centaines de mètres de profondeur.

\section{POUR UNE GESTION ADAPTÉE AUX SPÉCIFICITÉS DES GISEMENTS D'EAU PROFONDE}

La nature même des gisements profonds d'eau souterraine, leur contexte, leurs caractéristiques physiques et les propriétés physico-chimiques de l'eau imposent des contraintes particulières de gestion de cette ressource en eau.

Elles seront largement évoquées tout au long des exposés qui suivront, qu'ils soient relatifs aux techniques et méthodes d'étude, aux aspects quantitatifs, qualitatifs de leur utilisation, voire aux aspects réglementaires.

On se limitera donc à ne rappeler que les principales de ces caractéristiques et leur implication en matière d'exploitation et de gestion de l'eau.

- Les grandes profondeurs d'accès imposent des conditions techniques de foration particulières. Initialement issues des techniques pétrolières, on assiste depuis peu à l'adaptation de techniques jusqu'à présent limitées aux faibles profondeurs, telle la foration à l'air.

Quoiqu'il en soit les coûts restent élevés et imposent au maître d'ouvrage de minimiser les risques d'échecs, et donc d'éviter les objectif́s restreints ou discontinus dans l'espace (compartimentés, karstifiés), sans études préalables adaptées au contexte et aux enjeux: Jes méthodes d'études existent.

Il est a fortiori encore exceptionnel que ces gisements soient dotés de piézomètres de suivi, sauf à utiliser à cet effet des ouvrages d'exploitation hors service, ou le cas échéant, des reconnaissances pétrolières. Il faut citer la 
nappe infra-molassique de Midi-Pyrénées qui commence à être équipée de piézomètres spécifiques, mais aussi le suivi de l'albien en Ile-de-France. Il faut souligner l'intérêt qu'il y aurait à concevoir un suivi piézométrique de ces nappes profondes.

- Les grandes profondeurs de gisement peuvent conférer à l'eau souterraine des propriétés physico-chimiques positives ou négatives vis-à-vis des usages escomptés : minéralisation et température valorisée en thermalisme, température pour la valorisation géothermique, mais pouvant induire des propriétés corrosives, etc..

- Les grandes épaisseurs de recouvrement confèrent en principe aux gisements profonds une protection efficace contre les risques de pollution. Cependant, dans la mesure où ils affleurent (ce qui garantit leur alimentation) ils « prêtent le flan » à des contaminations possibles et difficilement remédiables. Ces zones d'affleurement de formations devenant profondes doivent donc être impérativement protégées lorsqu'on attend de cette ressource un usage qualitativement contraignant tel que l'AEP.

(Exception: les systèmes profonds à nappes libres vulnérables, des plateaux calcaires karstiques des Causses).

Malgré leur protection naturelle, les nappes profondes ne sont pas à l'abri de contamination par des ouvrages permettant, volontairement ou non, leur communication avec des nappes superficielles de qualité dégradée (ex. : Beauce). On recommandera donc que les ouvrages captant les nappes profondes soient parfaitement isolés des autres systèmes susjacents.

Le recouvrement protecteur des nappes profondes associé à des structures adéquates les prédisposent à des stockages spécifiques tels le gaz naturel.

\section{- Nappe profonde et nappe captive}

La tentation est grande d'assimiler les concepts de profondeur et de captivité des systèmes aquifères. Il est vrai que plus un système est profond, plus il a de chances d'être mis en charge.

C'est effectivement ce qu'on observe fréquemment

Exemple : calcaires de Beauce et de Sologne, libres à 60 $100 \mathrm{~m}$ de profondeur au Nord de la Loire, et devenant captifs vers le Sud, en Sologne; nappes du Jurassique et du Trias de Lorraine, etc...

Sur la cinquantaine de systèmes profonds identifiés au niveau national, le caractère indiscutablement captif dans leur zone d'exploitation principale est assuré pour une trentaine d'entre eux. Notons l'existence de systèmes profonds non captifs (notamment dans certains bassins péri-alpins) et de systèmes captifs non profonds (c'est-à-dire à moins de $200 \mathrm{~m}$ ) : sables landéniens des Flandres, craie et sables thanétiens du Nord du Bassin parisien, Dogger du Poitou...).

Le caractère captif facilite, bien sûr, l'exploitation en réduisant les hauteurs de refoulement par rapport aux profondeurs d'accès. Mais il garantit surtout au système une protection efficace contre des contaminations par des nappes superficielles. Il est donc souvent indispensable de veiller à maintenir cette charge (y compris en contrôlant son exploitation en zone périphérique à nappe libre). La piézométrie différentielle en est le moyen le plus efficace.

\section{DE LA MÉCONNAISSANCE À LA CONNAISSANCE DES GISEMENTS D'EAU PROFONDE}

Ce qui caractérise avant tout les eaux profondes, c'est l'extrême variabilité de leur état de connaissance.
En principe, plus une ressource est profonde, moins elle est utilisée, et moins elle est utilisée, moins elle est connue! En réalité il faut nuancer cette vision un peu réductrice.

La connaissance est effectivement essentiellement liée à l'exploitation. Très rares en effet sont, en France, les travaux spécifiquement dits « de reconnaissance hydrogéologique ». Quand bien même certaines études géophysiques ont lieu, c'est en vue de l'implantation d'ouvrage d'exploitation, c'est-à-dire financés par les exploitants. De telles études sont effectivement justement préconisées pour la recherche d'eau profonde en milieu discontinu (des Alpes du Sud par exemple) pour minimiser les risques d'échecs.

Remarquons que les «travaux de reconnaissance " sont plus couramment entrepris dans des pays à contexte de rareté de la ressource en eau, où des crédits publics peuvent leur être alloués (Afrique du Nord, Moyen Orient...). Ce cas est encore exceptionnel en France. Gageons qu'il le sera de moins en moins...

Autre exception à la règle : les reconnaissances pétrolières qui ont parfois contribué fortuitement à la connaissance des eaux profondes : exploitation de données géologiques, voire réutilisation de puits de reconnaissance. Fortuitement parce que tel n'était pas leur objectif initial ! Ainsi par exemple ils seront préférentiellement implantés sur des structures anticlinales alors que l'hydrogéologue préfère généralement les éviter...

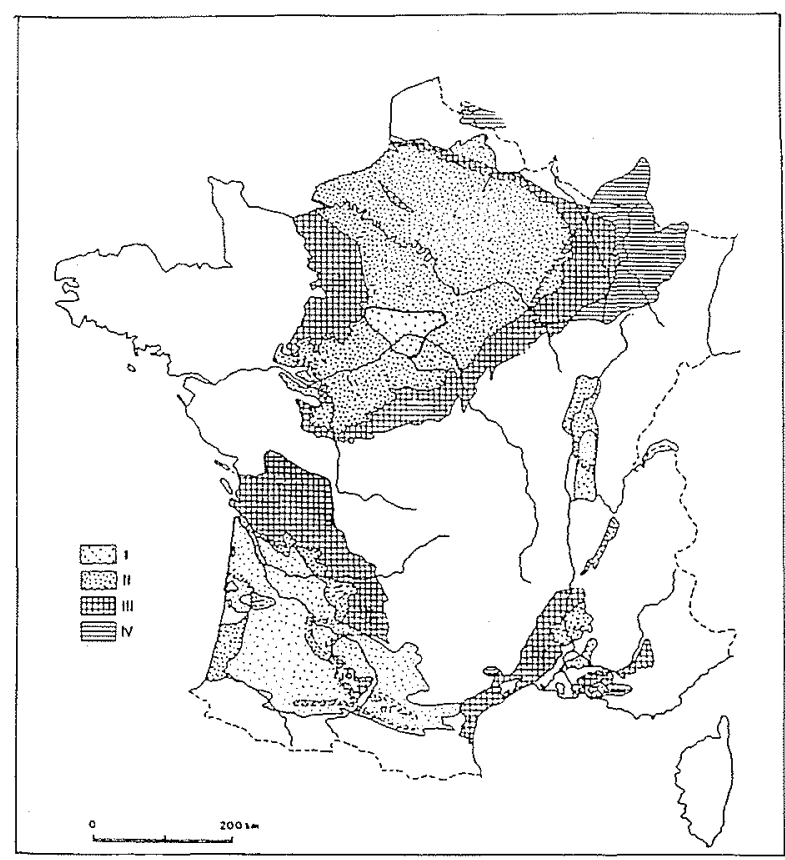

2. Gisements d'eau profonde de France métropolitaine : extension des grands domaines géologiques susceptibles d'être aquifères.

I $\quad 1^{\mathrm{er}}$ sous-sol : systèmes aquifères profonds des formations du Tertiaire.

II $2^{\mathrm{c}}$ sous-sol : systèmes aquifères profonds des formations du Crétacé.

III $3^{\mathrm{e}}$ sous-sol : systèmes aquifères profonds des formations du Jurassique.

IV $4^{\mathrm{e}}$ sous-sol : systèmes aquifères profonds des formations du Trias et du Primaire.

Concernant l'identification, l'extension et la géométrie des gisements, les grandes nappes profondes des bassins parisien et aquitain sont mieux connues que les gisements plus com- 
partimentés et discontinus structurellement et lithologiquement des zones de montagne, notamment du Jura aux Alpes et au Nord des Pyrénées. Toutefois ceci n'est pas toujours vrai sur le plan de la productivité : on observe encore des discontinuités surprenantes au niveau des productivités d'ouvrage captant des nappes profondes bien identifiées des grands bassins sédimentaires.

Par ,ailleurs, il est des nappes profondes paradoxalement mieux connues que certaines nappes superficielles susjacentes, parce que précisément plus exploitées que ces dernières. Malgré les coûts d'accès, l'eau y est en effet de qualité nettement meilleure, et conforme par exemple à l'usage AEP. C'est un cas de figure fréquent dans le bassin aquitain, où les nappes superficielles sont trop souvent rendues inaptes à un tel usage.

D'une manière plus générale, il faut malheureusement constater que le besoin de reconnaître et connaître les eaux profondes est minime dès lorsqu'une ressource superficielle, plus accessible, offre une alternative apparente. Toutefois au critère quantitatif devrait progressivement se substituer un critère qualitatif : les gisements profonds, bien mieux protégés de toute pollution diffuse, ponctuelle ou accidentelle, offrent un potentiel qualité encore largement sous-exploité. Dans la culture de l'exploitant, le traitement d'une eau superficielle visible apparaît encore trop souvent plus sécurisant que le captage d'une eau profonde de qualité, mais invi- sible et réputée aléatoire, bien qu'économiquement plus avantageuse.

Ce n'est évidemment pas le cas des « exploitants spécifiquement d'eaux profondes » qui recherchent leurs propriétés minérales et thermiques en vue d'une valorisation économique (thermalisme, embouteillage, AEP sans alternative, géothermie). On peut en outre imaginer que se développera de plus en plus fréquemment une compétition économique et technique entre le traitement d'eau superficielle vulnérable et le captage de ressources protégées, notamment profondes. Encore faudra-t-il que les données du problème soient connues et que, pour commencer, les gisements d'eau profonde soient mieux identifiés et caractérisés, surtout lorsque les ressources alternatives sont vulnérables et/ou menacées.

L'avenir se situera donc autant sur le plan technique : hydrogéologie, foration, exhaure..., qu'économique : analyses comparées d'alternatives, et relationnel : sensibilisation et information des différents acteurs institutionnels et sociaux, des usagers potentiels. Le cadre prévu par la loi sur l'eau de 1992 s'y prête. Les SDAGE ont souligné à la fois l'intérêt et la méconnaissance de ce potentiel

Il reste donc à trouver les moyens de remédier à cette inadéquation, avec une prise de conscience politique de cette nécessité, dès maintenant, c'est-à-dire avant que ne se multiplient les situations d'urgence.

Alors un jour s'apercevra-t-on peut-être que les nappes profondes ne sont pas si profondes que cela... 